\title{
Qualidade de vida de crianças e adolescentes asmáticos: sua relação com estratégias de enfrentamento materno
}

\author{
Quality of life of asthmatic children and adolescents: relation to maternal coping
}

Gimol Benzaquen Perosa1 , Isabel de Andrade Amato², Ligia Maria S. S. Rugolo ${ }^{3}$, Giesela Fleisher Ferrari', Maria Carolina F. A. de Oliveira ${ }^{5}$

\section{RESUMO}

Objetivo: Avaliar a qualidade de vida de crianças e adolescentes asmáticos, sua relação com variáveis sociodemográficas e clínicas e estratégias de enfrentamento materno.

Métodos: Estudo transversal no qual crianças e adolescentes com asma responderam a um questionário de qualidade de vida, e suas mães a uma escala de enfrentamento.

Resultados: Foram estudadas 42 crianças e adolescentes com idades entre 7 e 15 anos, sendo $74 \%$ classificados como tendo um quadro de asma persistente moderada/grave, $19 \%$ como persistente leve e $7 \%$ asma intermitente; $69 \%$ dos entrevistados apresentaram prejuízo na qualidade de vida, com escores médios variando de 4,7 a 3,5 e maior prejuízo no domínio sintomas (escore $=3,6$ ). Houve associação significativa entre escolaridade materna e índice geral de qualidade de vida, mas não entre gravidade da asma e tipo de enfrentamento materno. Grande parte das estratégias utilizadas pelas mães para enfrentar as crises do filho estava direcionada ao manejo de estressores ou práticas religiosas, estas com correlação negativa com o índice geral de qualidade de vida da criança, sinalizando que mães cujos filhos tinham pior qualidade de vida usavam mais enfrentamentos religiosos.

Conclusões: Crianças asmáticas, especialmente com asma persistente moderada/grave, apresentaram alterações significativas em sua qualidade de vida. A alta porcentagem de uso de estratégias religiosas por parte das mães, especialmente frente a quadros mais graves, parece indicar que elas se sentem impotentes para atuar, necessitando de orientações concretas e factíveis para uma população de baixa renda.

Palavras-chave: qualidade de vida; asma; enfrentamento; criança; adolescente.

\section{ABSTRACT}

Objective: To evaluate the quality of life of asthmatic children and adolescents, its relation with sociodemographic and clinical variables, and maternal coping strategies.

Methods: Cross-sectional study in which children and adolescents with asthma answered a quality of life questionnaire, and their mothers did the same with a coping scale.

Results: Out of the 42 children and adolescents investigated, $74 \%$ were classified as having mild/severe persistent asthma; $19 \%$, mild persistent asthma; and $7 \%$, intermittent asthma. A total of $69 \%$ of the participants showed impaired quality of life with mean scores ranging between 4.7 and 3.5, with greater harm in the domain of symptoms (score=3.6). There was a significant association between maternal schooling and the general index of quality of life, whereas maternal coping strategies were not associated with the severity of asthma. A large number of strategies used by
Instituição: Faculdade de Medicina de Botucatu da Universidade Estadual Paulista "Júlio de Mesquita Filho" (Unesp), Botucatu, SP, Brasil

'Doutora em Psicologia Social pela Pontifícia Universidade Católica de São Paulo (PUCSP); Professora-assistente doutora na Faculdade de Medicina de Botucatu da Unesp, Botucatu, SP, Brasil

${ }^{2}$ Médica no programa de residência da Faculdade de Medicina da Universidade Estadual de Campinas (Unicamp), Campinas, SP, Brasil

${ }^{3}$ Livre-Docente em Pediatria pela Faculdade de Medicina de Botucatu da Unesp; Professora adjunta da Faculdade de Medicina de Botucatu da Unesp, Botucatu, SP, Brasil

${ }^{4}$ Doutora em Pediatria pela Universidade de São Paulo (USP); Professora doutora da Faculdade de Medicina de Botucatu da Unesp, Botucatu, SP, Brasil ${ }^{5}$ Psicóloga do Serviço de Psicologia do Hospital das Clínicas da Faculdade de Medicina de Ribeirão Preto da USP, Ribeirão Preto, SP, Brasil
Endereço para correspondência:

Gimol Benzaquen Perosa

Campus de Rubião Junior

CEP 18618-970 - Botucatu/SP

E-mail: gimol@fmb.unesp.br

Fonte financiadora: Bolsa de Iniciação Científica da Fundação de Amparo à Pesquisa do Estado de São Paulo (Fapesp), processo 2008/58938-6 Conflito de interesses: nada a declarar

Recebido em: 4/9/2012

Aprovado em: 31/1/2013 
mothers to cope with their children's crises were related to the management of stressors or to religious practices, and the latter presented negative correlation with the children's quality of life general index, showing that mothers whose children had worse quality of life used more religious coping.

Conclusions: Asthmatic children, particularly those with moderate/severe persistent asthma, showed significant alterations as to quality of life. The high percentage of mothers using religious strategies, particularly in face of more severe clinical conditions, seem to indicate that they feel powerless to act, thus requiring concrete and useful orientation to low income families.

Key-words: quality of life; asthma; coping; child; adolescent.

\section{Introdução}

A prevalência da asma vem aumentando nas últimas décadas e, atualmente, é uma das principais doenças crônicas no mundo, atingindo crianças de todas as faixas etárias, de diferentes classes sociais e etnias ${ }^{(1,2)}$. Asma é um problema de Saúde Pública, com a necessidade de programar ações apropriadas para seu controle ${ }^{(3)}$.

A consequência de tal enfermidade em crianças não é apenas física, representada pelas dificuldades respiratórias, mas também causa forte impacto emocional, associado ao absenteísmo escolar, às restrições sociais, ao estresse, aos distúrbios afetivos, à depressão, à insônia e às percepções sociais negativas, que acabam alterando a qualidade de vida $(\mathrm{QV})$ da criança e de seus familiares ${ }^{(2,4)}$. A doença não tratada, além de impor limites às atividades físicas, pode tornar a criança insegura pelo caráter imprevisível das crises, quadro que se agrava por receber poucas informações sobre sua doença $a^{(5)}$. Quando as mães explicam às crianças os eventos estressantes relacionados à asma, os filhos lidam melhor com as crises, apresentam menos distúrbios de comportamento e melhor qualidade de vida ${ }^{(6,7)}$.

A asma, como outras doenças crônicas, é uma condição estressante não só para a criança, como também para seus pais. A forma de enfrentamento da família influencia na adesão da criança ao tratamento ${ }^{(8,9)}$ e tem um papel crítico na QV da criança. As habilidades da família em se adaptar às múltiplas demandas de uma criança com doença crônica não dependem apenas da gravidade do quadro, mas da capacidade da mãe em avaliar e reagir aos estressores ${ }^{(7)}$. Sales $e t$ al ${ }^{(4)}$, após ajuste à gravidade da asma e a estressores familiares recentes, demonstraram que mães utilizando enfrentamentos mais ativos para solucionar problemas tinham crianças com baixa ansiedade e melhor QV do que aquelas que aplicavam estratégias de esquiva.

A QV em saúde representa a percepção do paciente em relação à sua doença, aos sintomas e aos estados funcional, psicológico e social. É um componente essencial nas avaliações sobre o estado de saúde do paciente, sua resposta ao tratamento e a progressão do quadro ${ }^{(10)}$. As primeiras tentativas de avaliação da QV de crianças foram realizadas a partir da percepção dos pais, pelo fato de se considerar que as crianças eram incapazes de avaliar seus ganhos e perdas. Estudos posteriores mostraram que as crianças são capazes de compreender o processo saúde-doença ${ }^{(11)}$, e que filhos e pais nem sempre compartilham a mesma visão sobre o impacto da doença em suas vidas ${ }^{(10,12)}$. Assim, a tendência atual é utilizar instrumentos respondidos por crianças a fim de obter suas próprias percepções sobre a doença, bem como envolvê-las em decisões quanto ao seu tratamento e cuidados ${ }^{(12,13)}$.

Nesta perspectiva, Juniper et al ${ }^{(14)}$ elaboraram um questionário para participantes com mais de sete anos, o Pediatric Asthma Quality of Life Questionnaire (PAQLQ), a partir de sua experiência com o Asthma Quality of Life Questionnaire (AQLQ), para adultos. O PAQLQ foi validado para a língua inglesa e posteriormente publicado em outros 20 idiomas. Tem como vantagens ser aplicável mesmo quando o quadro de asma está estável, conseguir captar pequenas mudanças e incluir funções fundamentais à vida das crianças ${ }^{(14)}$. A primeira versão para a língua portuguesa foi efetuada por La Scala et a $l^{(13)}$. Em 2010, Sarria et a $l^{(15)}$ apresentaram uma nova versão e determinaram as propriedades psicométricas da versão oficial para o português, com bom desempenho psicométrico e adequação do instrumento ao contexto brasileiro.

O objetivo do presente estudo foi avaliar diretamente crianças e adolescentes asmáticos quanto a sua qualidade de vida, relacionando-a com algumas variáveis sociodemográficas, clínicas e com estratégias de enfrentamento materno. Partiu-se das hipóteses de que: crianças e adolescentes com quadros mais graves têm pior qualidade de vida, há associação entre a gravidade da asma e os modos de enfrentamento materno e destes com a QV da criança e do adolescente.

\section{Método}

Estudo transversal de crianças com asma acompanhadas em um Ambulatório de Pneumologia Pediátrica de um hospital-escola do interior paulista, no período de fevereiro 
a junho de 2009. O estudo foi aprovado pelo Comitê de Ética em Pesquisa da Instituição, e participantes e seus responsáveis assinaram o termo de consentimento livre e esclarecido.

Os pacientes foram selecionados no dia da consulta médica no Ambulatório Especializado e foram incluídos aqueles que preencheram os seguintes critérios de inclusão: idade maior ou igual a sete anos, sem comorbidades respiratórias, como fibrose cística ou displasia broncopulmonar, e que não estivessem usando corticoide sistêmico nas duas semanas prévias ao estudo. Dentre os selecionados que preencheram os critérios de inclusão, em cada dia de atendimento foram sorteados três pacientes, por ser este o número máximo para viabilizar a avaliação no mesmo dia da consulta. As crianças que não conseguiram responder ao questionário, devido à dificuldade de entendimento ou indisposição clínica, foram excluídas da avaliação de QV; porém, suas mães foram mantidas no estudo para avaliar os modos de enfrentamento. A análise das mães e crianças ocorreu em ambiente de privacidade, com duração média de 50 minutos.

A classificação da gravidade da asma foi efetuada pela equipe médica, conforme proposto pelas IV Diretrizes Brasileiras para o Manejo da Asma, de $2006^{(16)}$, levando-se em conta os seguintes critérios: presença de sintomas diurnos e noturnos; limitação da atividade física; necessidade do uso de medicação de resgate; função pulmonar; e frequência das exacerbações. Segundo tais critérios, os pacientes foram classificados como tendo asma intermitente, persistente leve e persistente moderada/grave.

Foi solicitada autorização para usar o instrumento de QV diretamente aos autores ${ }^{(14)}$, que enviaram o material traduzido para o português por La Scala $e t a l^{(13)}$.

O PAQLQ é um instrumento específico para avaliar a $\mathrm{QV}$ em indivíduos portadores de asma, com idades entre 7 e 17 anos. É composto por 23 itens distribuídos em três domínios: limitações de atividade (5 itens), sintomas (10 itens) e função emocional (8 itens). Todos os itens do PAQLQ são respondidos em escala Likert de sete pontos, que varia de um (gravemente afetado) a sete (não afetado). Em seguida são somados, e suas médias representam os escores (total e por domínio).

A Escala Modos de Enfrentamento de Problemas (EMEP) foi validada para a população brasileira por Seidl et al ${ }^{(17)}$. É composta por 45 itens, distribuídos em quatro fatores de enfrentamento: centrado no problema $(\alpha=0,84)$; focalizado na emoção $(\alpha=0,81)$; busca de práticas religiosas/pensamento fantasioso $(\alpha=0,74)$; e busca de suporte social $(\alpha=0,70)$.
As respostas são fornecidas em escala Likert de cinco pontos. Escores mais elevados são indicativos de maior utilização de determinada estratégia de enfrentamento.

As informações sociodemográficas da criança e de seus familiares, incluindo idade materna e da criança, nível de escolaridade e ocupação, foram obtidas dos prontuários médicos. O desfecho de interesse foi a QV das crianças e dos adolescentes asmáticos.

O banco de dados foi estruturado no software Excel 2000 e as análises estatísticas efetuadas pelo programa SAS for Windows, versão 9.2. Foi aplicado o teste de Shapiro-Wilk para checar a normalidade das variáveis. Como os escores de QV apresentaram distribuição normal, a comparação das médias dos diferentes domínios foi efetuada por análise de variância (ANOVA) para medidas repetidas, seguida do teste de comparações múltiplas de Tukey. Para comparar os modos de enfrentamento, como os escores não tiveram distribuição normal, foram ajustados um modelo linear generalizado Gama e uma função de ligação log, considerando medidas repetidas (equação de estimação generalizada). A seguir, aplicou-se um teste de comparações múltiplas (LSMeans test) com $p<0,05$, comparando as medianas $2 \times 2$. Para avaliar possíveis preditores do índice geral de QV, dentre as variáveis sociodemográficas e clínicas, calculou-se uma regressão logística univariada. A associação entre modos de enfrentamento materno e a QV da criança foi investigada pela correlação de Pearson. Em todas as análises, o nível de significância foi $5 \%$.

\section{Resultados}

Do total de 58 sujeitos aleatoriamente selecionados, oito mães se recusaram a participar por medo de perder o transporte; cinco crianças não conseguiram responder ao questionário; e três avaliações foram suspensas por indisposição clínica da criança. No total, 42 crianças responderam ao questionário de QV e 50 mães às formas de enfrentamento.

A idade média das crianças foi de 9,5 anos, variando de 7 a 15 , com um predomínio do sexo feminino $(\mathrm{n}=25 ; 59 \%)$. A maioria $(\mathrm{n}=31 ; 74 \%)$ foi classificada como tendo asma persistente moderada/grave, oito (19\%) pacientes tinham asma persistente leve, e poucos participantes apresentavam asma intermitente $(\mathrm{n}=3 ; 7 \%)$.

A idade média das mães foi de 34 anos, 22 (44\%) tinham entre 18 e 30 anos e 3 tinham mais de 50 . Quanto à escolaridade, 24 (48\%) não concluíram o ensino fundamental 
e $19(38 \%)$ completaram o ensino médio. Apenas quatro mães $(8 \%)$ contavam com ensino fundamental ou superior completos. A maioria possuía um parceiro fixo $(n=41 ; 82 \%)$ e $25(50,0 \%)$ eram donas de casa.

Quanto à QV das crianças, a média do índice geral e dos diferentes domínios variou de 4,7 a 3,5, com prejuízo significativo no domínio sintomas, quando comparado com limitação de atividades e função emocional (Tabela 1).

Entre as variáveis sociodemográficas e clínicas, apenas a escolaridade materna mostrou-se fator de proteção: filhos de mães com maior grau de escolaridade tinham $56 \%$ mais chance de ter bom índice geral de QV (Tabela 2).

Ao comparar os modos de enfrentamento materno, verificou-se que o emocional foi significativamente menos utilizado quando comparado aos centrados no problema, religioso e de apoio social (Tabela 3). Ao correlacionar os tipos de enfrentamento, houve correlação significativa somente entre enfrentamento religioso e emocional $(0,007 \%)$.

Não houve associação entre a gravidade da asma e o modo de enfrentamento materno (Tabela 4), porém, houve correlação negativa entre os escores médios do enfrentamento religioso com o índice geral de QV e com o domínio sintomas (Tabela 5).

\section{Discussão}

Neste estudo, houve predomínio de crianças/adolescentes com quadros moderados/graves, o que é esperado na casuística de um centro terciário, com maior porcentagem de casos graves em relação à registrada em estudos populacionais, quando predominam aqueles intermitentes ou persistentes leves ${ }^{(1)}$.

A maioria das crianças estudadas apresentou alteração na QV, com índice médio geral de 4,0 e valores médios entre três e quatro nos diferentes domínios do PAQLQ. Pacientes com asma intermitente tiveram pontuações mais altas (escore =5,6), isto é, melhor QV, quando comparados àqueles com persistente leve $($ escore $=4,0)$ e persistente moderada/grave (escore $=3,8)$. Segundo Vidal et $a l^{(9)}$, a pontuação abaixo de 5,0 traduz alteração significativa na QV. Esses autores avaliaram, pelo PAQLQ, crianças chilenas com quadros de asma persistente em diferentes níveis de gravidade, e obtiveram pontuação menor que 5,0 em 40\%

Tabela 1 - Média e desvio padrão dos escores da qualidade de vida total e dos domínios atividades, sintomas e emoções de 42 crianças asmáticas submetidas ao PAQLQ-A

\begin{tabular}{lcc}
\hline Domínios & Média & DP \\
\hline Índice geral & 4,12 & 1,27 \\
Limitação nas atividades & 4,64 & 1,48 \\
Sintomas & $3,58^{\star}$ & 1,69 \\
Função emocional & 4,49 & 1,59 \\
\hline
\end{tabular}

*diferença significante entre sintomas e os demais domínios (Anova; $p<0,05$ e Tukey; $p<0,05)$; DP: desvio padrão.

Tabela 2 - Modelo de regressão logistica univariada para avaliar preditores do índice de qualidade de vida de crianças com asma

\begin{tabular}{lccc}
\hline Variáveis & Valor $\boldsymbol{p}$ & OR & IC95\% \\
\hline $\begin{array}{l}\text { Ocupação } \\
\text { materna }\end{array}$ & 0,85 & 1,05 & $(0,66-1,66)$ \\
Idade materna & 0,43 & 0,83 & $(0,53-1,32)$ \\
$\begin{array}{l}\text { Escolaridade } \\
\text { materna }\end{array}$ & 0,02 & 0,56 & $(0,34-0,90)$ \\
$\begin{array}{l}\text { Estado civil } \\
\text { Gravidade da }\end{array}$ & 0,54 & 1,2 & $(0,66-2,20)$ \\
doença & 0,51 & 1,19 & $(0,70-2,00)$ \\
\hline $\mathrm{n}=42 ;$ OR: Odds Ratio; IC95\%: intervalo de confiança de $95 \%$.
\end{tabular}

Tabela 3 - Mediana, percentis 75 e 25 dos modos de enfrentamento a que recorriam as 50 mães de crianças asmáticas

\begin{tabular}{lccc}
\hline Modos de enfrentamento & Med. & P25 & P75 \\
\hline Problema & 3,55 & 3,30 & 4,00 \\
Religioso & 3,70 & 2,75 & 4,40 \\
Apoio social & 3,30 & 2,60 & 4,20 \\
Emocional & $1,80^{*}$ & 1,40 & 2,58 \\
\hline
\end{tabular}

*diferença significante entre enfrentamento emocional e os demais modos (equação de estimação generalizada, seguida do teste de comparações múltiplas; $p<0,05$ ); Med.: mediana.

Tabela 4 - Comparação entre os modos de enfrentamento das mães de crianças com asma moderada/grave e intermitente/ persistente leve

\begin{tabular}{lccccccc}
\hline \multirow{2}{*}{$\begin{array}{l}\text { Modos de } \\
\text { enfrentamento }\end{array}$} & \multicolumn{3}{c}{$\begin{array}{c}\text { Mães de crianças com asma } \\
\text { moderada/grave }\end{array}$} & \multicolumn{3}{c}{$\begin{array}{c}\text { Mães de crianças sem asma } \\
\text { intermitente/persistente leve }\end{array}$} & \multirow{2}{*}{ Valor $\boldsymbol{p}^{*}$} \\
\cline { 2 - 7 } & Mediana & $\mathbf{P 2 5}$ & $\mathbf{P 7 5}$ & Mediana & $\mathbf{P 2 5}$ & $\mathbf{P 7 5}$ & \\
\hline Problema & 3,55 & 3,30 & 4,00 & 3,50 & 2,80 & 4,40 & 0,556 \\
Religioso & 3,65 & 2,20 & 4,40 & 3,70 & 3,10 & 4,00 & 0,747 \\
Apoio social & 3,30 & 2,60 & 4,20 & 2,60 & 2,00 & 3,40 & 0,135 \\
Emocional & 1,80 & 1,30 & 2,50 & 1,70 & 1,60 & 2,50 & 0,979 \\
\hline
\end{tabular}

$\mathrm{n}=50$; *equação de estimação generalizada para localizar as diferenças entre os diversos modos de enfrentamento. 
Tabela 5 - Correlação entre os modos de enfrentamento materno e os escores do índice geral e dos três domínios de qualidade de vida $(n=42)$

\begin{tabular}{lccccc}
\hline $\begin{array}{l}\text { Modos de } \\
\text { enfrentamento }\end{array}$ & Valores & $\begin{array}{c}\text { Índice } \\
\text { geral }\end{array}$ & $\begin{array}{c}\text { Limitação de } \\
\text { atividades }\end{array}$ & Sintomas & $\begin{array}{c}\text { Função } \\
\text { emocional }\end{array}$ \\
\cline { 2 - 5 } Problema & $r$ & $-0,129$ & $-0,259$ & 0,160 & 0,043 \\
\multirow{2}{*}{ Religioso } & $p$ & 0,412 & 0,096 & 0,310 & 0,782 \\
& $r$ & $-0,538$ & $-0,002$ & $-0,375$ & $-0,232$ \\
Apoio social & $p$ & $<0,001$ & 0,988 & 0,014 & 0,138 \\
\multirow{2}{*}{ Emocional } & $r$ & 0,026 & 0,034 & 0,026 & $-0,003$ \\
& $p$ & 0,869 & 0,828 & 0,868 & 0,982 \\
& $r$ & $-0,231$ & 0,095 & $-0,176$ & $-0,198$ \\
\hline
\end{tabular}

$r$. coeficiente de correlação de Pearson; $p$ : significância estatística.

delas. Resultado semelhante, com índice médio geral de 4,6, foi obtido por Levy et al ${ }^{(18)}$ em crianças e jovens com idades entre 4 e 17 anos.

No Brasil, crianças avaliadas em serviços terciários com o mesmo instrumento mostraram melhor percepção de sua QV, com pontuações médias em torno de 5,0 e 6,0 ${ }^{(13,15)}$. Algumas hipóteses podem ser levantadas para explicar os diferentes resultados. Em primeiro lugar, nesta pesquisa foram incluídas várias crianças com quadros de asma persistente grave controlada, possivelmente com pior QV, excluídas no estudo de Sarria et al ${ }^{(15)}$ e pouco frequentes (apenas duas) no de La Scala et al ${ }^{(13)}$.

Deve-se considerar também que apesar dos sujeitos dos três estudos serem pacientes atendidos em serviços terciários, há diferenças na clientela dos três centros. Dois deles estão localizados em capitais, e este estudo foi desenvolvido em uma instituição situada em cidade de médio porte, que atende pacientes de uma ampla região composta de cidades médias, pequenas e comunidades rurais. Está documentado que os portadores de asma residentes em comunidades rurais têm mais dificuldade de acesso aos serviços de saúde, principalmente de urgência, com taxas mais altas de mortalidade ${ }^{(19,20)}$. Nesta pesquisa, os sujeitos moradores de pequenas localidades podem ter vivenciado crises mais prolongadas devido à falta de recursos próximos ao domicílio, provocando uma percepção mais negativa de sua $\mathrm{QV}$.

As crianças consideraram o domínio sintomas como sendo o que acarretava mais prejuízos à QV. O mesmo foi observado em estudo com crianças portadoras de quadros de asma persistente leve e moderada ${ }^{(21)}$. Em pacientes adultos com asma moderada/grave, antes de iniciar o tratamento, falta de ar, sibilos e tosse foram fortes preditores de pior $\mathrm{QV}^{(22)}$.
O domínio emocional, assim como em outras pesquisas que tiveram crianças como sujeitos ${ }^{(9,13,18)}$, e diferentemente dos adultos ${ }^{(22)}$, foi percebido como o menos prejudicado. Os resultados quanto à repercussão emocional da doença crônica em crianças são controversos. Há estudos em que as mesmas apresentaram medo, ansiedade e depressão, enquanto em outros não foram verificados prejuízos emocionais, principalmente nas menores ou com tempo maior de convívio com a doença ${ }^{(23)}$. Para alguns autores, baixos níveis de ansiedade e depressão, especialmente nos quadros graves, podem sinalizar um perfil altamente defensivo, o estilo repressivo adaptativo, como se a criança, para se adaptar ao quadro, subestimasse ou escondesse os sintomas e sua ansiedade, o que acaba repercutindo negativamente em sua saúde, com sinais de tensão, dores de cabeça, alergias, úlcera e hipertensão ${ }^{(24)}$.

Entre os fatores sociodemográficos, apenas a escolaridade do cuidador mostrou-se preditora de melhor índice de QV. Em outro estudo, com crianças italianas, a baixa escolaridade dos pais foi a variável com maior força preditiva sobre a prevalência de quadros de asma, especialmente de asma grave e de hospitalizações ${ }^{(19)}$.

A alta escolaridade materna tem sido associada com a capacidade de cuidar adequadamente, com a maior valorização do conhecimento científico e capacidade de articular recursos em prol das necessidades da criança ${ }^{(25)}$. Possivelmente, mães com mais escolaridade têm mais conhecimentos quanto à asma e sua evolução, sobre o que fazer quando os sintomas aparecem e como prevenir as crises, minimizando os prejuízos à QV da criança. Reforçando esta hipótese, na pesquisa de Stephan e Costa ${ }^{(26)}$ as mães mais pobres, com baixa escolaridade e as mais jovens foram as que possuíam menos conhecimento sobre o manejo adequado 
das exacerbações, reconhecimento do agravamento dos episódios de sibilância e dos fatores desencadeantes. A falta de conhecimento possivelmente retarda o reconhecimento das formas mais leves da doença, e as mães procuram os serviços médicos apenas frente a quadros graves, muitas vezes com necessidade de hospitalização ${ }^{(19)}$.

O grau de instrução também tem sido utilizado como indicador do nível socioeconômico ${ }^{(27)}$. O papel do mesmo na QV de crianças asmáticas ainda é controverso. Houve associação significativa em algumas pesquisas ${ }^{(19,27)}$, mas em outras não ${ }^{(15,28)}$. Pertencer à classe social baixa muitas vezes é indicativo da exposição do sujeito a vários fatores etiológicos de risco, como moradias com ambientes úmidos e mofo, presença de vários alérgenos, ser fumante passivo e ter maior chance de exposição à poluição externa, o que pode desencadear os quadros de asma. A falta de recursos financeiros, por sua vez, pode dificultar o acesso a serviços de saúde e à aquisição da medicação necessária para controlar as crises ${ }^{(18)}$. No entanto, é preciso ressaltar que embora o baixo nível socioeconômico seja um potencial fator de risco, quando a comunidade dispunha de estrutura adequada de atenção à saúde essa variável pouco influiu na incidência e na gravidade da doença ${ }^{(15)}$.

Com relação às estratégias de enfrentamento, contrariando a hipótese inicial do estudo, não houve associação entre gravidade do quadro e modo de enfrentamento materno. Tanto as mães de crianças com asma moderada/grave quanto aquelas com asma intermitente/leve utilizaram predominantemente práticas religiosas, seguidas de estratégias centradas no problema. Os enfrentamentos focalizados na emoção foram menos utilizados por todas elas.

A constatação de que as cuidadoras recorriam com alta frequência às estratégias direcionadas ao manejo do problema e a poucas estratégias focalizadas na emoção confirma dados de outros estudos, com pacientes adultos ${ }^{(29)}$, familiares de crianças com doenças crônicas ${ }^{(30)}$ e mães de crianças asmáticas $^{(4,31)}$. Estes identificaram o uso de estratégias ativas associado à baixa ansiedade e à melhor QV do filho.

Em relação à utilização de enfrentamentos religiosos, pesquisas anteriores já haviam sinalizado sua larga utilização por usuários dos serviços de saúde. Estudo de revisão mostrou que, na cultura brasileira, a maioria dos pacientes relatava crenças religiosas e acreditava que elas podiam ajudar a lidar com suas doenças ${ }^{(32)}$. Considera-se um padrão positivo de enfrentamento quando as práticas religiosas são acompanhadas por enfrentamentos centrados no problema, significando que o indivíduo, além de ter ações concretas para aliviar os sintomas, reza para evitar maior estresse. Entretanto, tal associação não foi verificada neste estudo. Os enfrentamentos religiosos se associaram aos emocionais e à pior QV da criança.

Enfrentamentos religiosos e centrados na emoção são utilizados por pacientes em estágios avançados da doença ${ }^{(32)}$, podendo significar que, frente a quadros mais graves, as mães tinham percepção da falta de controle do problema, esperavam milagres e, com isso, aliviavam a angústia. Se em curto prazo esses enfrentamentos ajudam a preservar o equilíbrio psicológico materno, seus efeitos em longo prazo preocupam por poderem colocar em risco a adesão ao tratamento ${ }^{(4)}$.

Algumas limitações do estudo precisam ser apontadas. Não foram investigadas outras características biológicas, exceto a gravidade da asma. Com relação à avaliação da severidade dos quadros, esta foi obtida indiretamente a partir do prontuário médico, quando da chegada ao serviço. Apesar de os médicos terem larga experiência em diagnóstico e terem utilizado o mesmo referencial para classificar a gravidade, não foi possível calcular o grau de confiabilidade entre os diferentes avaliadores.

A casuística foi pequena, porém, obtida de forma aleatória, por sorteio, sendo limitado o número de três crianças por consulta a fim de evitar a necessidade de retorno ao serviço para participação na pesquisa. Quanto à composição da amostra, devido às características do serviço foi obtida uma amostra com predomínio dos quadros de asma persistente moderada/grave. No entanto, com a amostra estudada foi possível responder às questões que motivaram a pesquisa.

Concluindo, crianças com asma, especialmente com quadros persistentes moderados/graves, apresentaram alterações em sua QV. A escolaridade materna mostrou-se fator protetor, enquanto a gravidade do quadro não influenciou a $\mathrm{QV}$, nem se associou às estratégias de enfrentamento materno. $\mathrm{O}$ enfrentamento religioso correlacionou-se com o índice geral de $\mathrm{QV}$ e com a sintomatologia da criança, indicando que as mães que usavam mais enfrentamentos religiosos tinham filhos com piores sintomas e índices gerais de QV.

A alta porcentagem do uso exclusivo de estratégias religiosas por parte de mães de filhos com sintomatologia mais grave sugere que as mesmas se sentem impotentes para atuar, e se beneficiariam de orientações concretas e factíveis para uma população de baixa renda. $\mathrm{Na}$ impossibilidade de controlar o quadro, elas deveriam se dirigir ao serviço de urgência mais próximo. Intervenções imediatas ajudam a reduzir a duração das crises e os prejuízos impostos à criança, os quais, em última estância, repercutem na QV da criança e de sua família. 


\section{Referências bibliográficas}

1. Simões SM, Cunha SS, Barreto ML, CruzAA. Distribution of severity of asthma in childhood. J Pediatr (Rio J) 2010;86:417-23.

2. Solé D, Wandalsen GF, Camelo-Nunes IC, Naspitz CK; ISAAC Brazilian Group. Prevalence of symptoms of asthma, rhinitis, and atopic eczema among Brazilian children and adolescents identified by the International Study of Asthma and Allergies in Childhood (ISAAC): phase 3. J Pediatr (Rio J) 2006;82:341-6.

3. Chatkin MN, Menezes AM. Prevalence and risk factors for asthma in schoolchildren in southern Brazil. J Pediatr (Rio J) 2005;81:411-6.

4. Sales J, Fivush R, Teague G. The role of parental coping in children with asthma's psychological well-being and asthma-related quality of life. J Pediatr Psychol 2008;33:208-19.

5. Tates K, Meeuwesen L. Doctor-parent-child communication. A (re)view of the literature. Soc Sci Med 2001;52:839-51.

6. Fivush R, Sales JM. Coping, attachment, and mother-child narratives of stressful event. Merrill Palmer Q 2006;52:125-51.

7. Kaugars AS, Klinnert MD, Bender BG. Family influences on pediatric asthma. J Pediatr Psychol 2004;29:475-91.

8. Castro EK, Piccinini CA. Implications of physical chronic disease in childhood to Family relationships: some theoretical questions. Psicol Reflex Crit 2002;15:625-35.

9. Vidal GA, Duffau TG, Ubilla PC. Calidad de vida en el niño asmático y su cuidador. Rev Chil Enf Respir 2007;23:160-6.

10. Ungar WJ, Mirabelli C, Cousins M, Boydell KM. A qualitative analysis of a dyad approach to health-related quality of life measurement in children with asthma. Soc Sci Med 2006;63:2354-66.

11. Perosa GB, Gabarra LM. Explanations proffered by children hospitalized due to illness: implications for communication between healthcare professional and patients. Interface Comunic Saude Educ 2004;8:135-47.

12. Eiser E, Morse R. Quality-of-life measures in chronic diseases of childhood. Health Technol Assess 2001;5:1-157.

13. La Scala CS, Naspitz CK, Solé D. Adaptation and validation of the pediatric asthma quality of life questionnaire (PAQLQ-A). J Pediatr (Rio J) 2005;81:54-60.

14. Juniper EF, Guyatt DH, Feeny DH, Ferrie PJ, Griffith LE, Townsend M. Measuring quality of life in children with asthma. Qual Life Res 1996;5:35-46.

15. Sarria EE, Rosa RC, Fischer GB, Hirakata VN, Rocha NS, Mattiello R. Fieldtest validation of the Brazilian version of the paediatric asthma quality of life questionnaire. J Bras Pneumol 2010;36:417-24.

16. Sociedade Brasileira de Pneumologia e Tisiologia. IV Diretrizes Brasileiras para o Manejo da Asma 2006. J Bras Pneumol 2006;32 (Suppl 7):S447-74.

17. Seidl EM, Tróccoli BT, Zannon CM. Factorial analysis of a coping measure. Psic Teor Pesq 2001;17:225-34.
18. Levy JI, Welker-Hood LK, Clougherty JE, Dodson RE, Steinbach S, Hynes HP et al. Lung function, asthma symptoms, and quality of life for children in public housing in Boston: a case-series analysis. Environ Health 2004;3:13.

19. Cesaroni G, Farchi S, Davoli M, Forastiere F, Perucci CA. Individual and areabased indicators of socioeconomic status and childhood asthma. Eur Respir J 2003;22:619-24.

20. Chrischilles E, Ahrens R, Kuehl A, Kelly K, Thorne P, Burmeister L et al. Asthma prevalence and morbidity among rural lowa schoolchildren. J Allergy Clin Immunol 2004;113:66-71.

21. Sancho ML, Herrera MR, Orellana JC. Impacto del asma en la edad pediatrica en la calidad de vida del niño. Alerg Inmunol Clin 2005;22:13-23.

22. Moy ML, Israel E, Weiss ST, Juniper EF, Dubé L, Drazen JM et al. Clinical predictors of health-related quality of life depend on asthma severity. Am J Respir Crit Care Med 2001;163:924-9.

23. Burke P, Elliott M. Depression in pediatric chronic illness: a diathesis-stress model. Psychosomatics 1999;40:5-17.

24. Phipps S, Steele RG, Hall K, Leigh L. Repressive adaptation in children with cancer: a replication and extension. Health Psychol 2001;20:445-51.

25. Engle PL, Menon P, Haddad L. Care and nutrition: concepts and measurement. Washington: International Food Policy Research Institute; 1997.

26. Stephan AM, Costa JS. Mothers of children with asthma's knowledge on the condition, in area covered by the family health program. Rev Bras Epidemiol 2009;12:671-9.

27. Von Rueden U, Gosh A, Rajmil L, Beisegger CE, Ravens-Sieberer U. Socioeconomic determinants of health related quality of life in childhood and adolescence: results from an European study. J Epidemiol Community Health 2006;60:130-5.

28. Solé D, Camelo-Nunes IC, Wandalsen GF, Mallozi MC, Naspitz C; Brazilian ISAAC's Group. Is the prevalence of asthma and related symptoms among Brazilian children related to socioeconomic status? J Asthma 2008;45:19-25.

29. Seidl EM, Rossi WS, Viana KF, Meneses AK, Meireles E. Children and adolescents living with HIVIAids and their families: psychosocial aspects and coping. Psic Teor Pesq 2005;21:279-88.

30. Schmidt C, Dell'Aglio DD, Bosa CA. Coping strategies of mothers of autistic children: dealing with behavioral problems and emotions. Psicol Reflex Crit 2007;20:124-31.

31. Mendonça MB, Ferreira EA. Adherence to treatment by caregivers of children with asthma. Rev Bras Cresc Desenv Hum 2005;15:56-68.

32. Faria JB, Seidl EM. Religiosity, coping and well-being in people living with HIV/ AIDS. Psicol Estud 2006;11:155-64. 